\title{
ANTIBACTERIAL ACTIVITY OF GERANIUM PRATENSE PREPARATIONS
}

\section{(C) Burakova M. A., Karavaeva A.V, Kauhova I. E., Razaryonova K. N., Zhokhova E. V.}

\section{St. Petersburg State Chemical-Pharmaceutical Academy, Russia}

Geranium pratense is a perennial herb with horizontal rootstock, palmately lobed leaves and pentamerous zygomorphic bluish flowers, widespread in Russia. The chemical composition of the aerial part and root part of $G$. pratense includes hydrolysable and condensed tannins, phenolic acids, flavonoids. Herb, rhizome and root of $G$. pratense in powder or by infusion or decoction are reportedly used in folk medicine in treatment of periodontal diseases and stomatitis. The aim of the present investigation was to determine optimal conditions for extraction of biologically active compounds from $G$. pratense, to evaluate antimicrobial activity of powdered extracts obtained from its aerial part and root part and to validate scientifically its use in traditional medicine. The plant matter (herb or rhizome and root of $G$. pratense, collected in 2010-2011 in Northwest Russia) was extracted with $40 \%$ ethanol. Trials varying in methods of extraction, particle size, extraction time and solvent to solid ratio were performed. The quantitative evaluation of tannins and flavonoids in $40 \%$ ethanol aqueous extracts and powdered extracts was carried out by spectrophotometric method. Results of assays were reported in gallic acid equivalents for tannins and rutin equivalents for flavonoids. Broth dilution method followed by subculturing to agar media without antibacterial agent was employed to assess minimal inhibition concentration (MIC) and minimal bactericidal concentration (MBC). Optimal conditions for extraction of phenolic constituents from raw material were evaluated. Powdered herb extract (PHE) was reported to contain $33.05 \%$ of tannins and $9.74 \%$ of flavonoinds. Antimicrobial studies suggested that PHE had inhibitory effect against $S$. aureus and $B$. cereus: the MIC was found to be $2 \mathrm{mg} / \mathrm{mL}$ against $S$. aureus, the MIB against $B$. cereus was $1 \mathrm{mg} / \mathrm{mL}$. The total content of tannins in powdered extract of $G$. pratense root part (PRE) is $41.56 \%$. PRE exhibited growth inhibitory activity against $S$. aureus, $B$. cereus and $P$. aeruginosa with MIB value of $0.5 \mathrm{mg} / \mathrm{mL}, 1 \mathrm{mg} / \mathrm{mL}$ and MIC value of $2 \mathrm{mg} / \mathrm{mL}$ respectively. PHE and PRE revealed antimicrobial activity against Gram-positive bacteria. The existence of microorganisms in particular Streptococcus sp. in an oral biofilm and dental plaque has profound implications for the etiology of periodontal disease. So the results indicate that the studied G. pratense preparations could become a new effective agent in a wide range of oral care products used in treatment-and-profilactic of periodontitis, gingivitis and stomatitis.

\section{DEVELOPMENT OINTMENT AND SUPPOSITORY WITH HYPERICUM PERFORATUM HERB POLYEXTRACT}

\section{() Teslev A. A., Vainshtein V. A., Kaukhova I.Ye., Tsygankova M. I., Hazzaa I. H.}

\section{St. Petersburg State Chemical-Pharmaceutical Academy, Russia}

Hypericum perforatum herb (John's wort) contains a variety of biologically active substances (BAS), such as hypericin, pseudohypericin, hyperforin, flavonoids, carotenoids, vitamins, etc. Great "ensemble" of St. John's wort's BAS causes a wide range of its therapeutic action. There are not technologies a soft pharmaceutical form with hydroalcoholic and oil extracts of the Hypericum perforatum herb. The aim of the study is to develop the technology the composition of ointments and suppositories with St. John's wort herb extracts in the amount of 1:1 or more in dosage forms. The first stage is the extraction of dry St. John's wort herb by solvents with increasing polarity - acetone, aqueous solutions of different concentrations of ethanol. In this case chlorophylls, anthracene derivatives, flavonoids are extracted. Liquid extracts were pooled and evaporated in the rotary vacuum evaporator until one-third of the volume. The components of ointment or suppository bases (COSB) (there are oils, polyethylene oxide, emulsifiers: MHD, tween-80, apian wax, witepsol etc.) were added in the evaporator flask. The total amount of the COSB was selected according to the source of plant material to obtain the desired module of the extract $-1: 1$ or 1:2. The evaporation was stopped when the distillation of volatile fraction was over. The residue after distillation of the extractant is a thick mass with soft or solid consistency, which can be easily melted and added into the ointments or suppositories. The content in the extract anthracenederivarives COSB was from 30 to $130 \mathrm{mg} \%$, and flavonoids - from 0.30 to $2.0 \%$ depending on the module of extraction. 